\title{
OPERATING CORE OF AN ORGANIZATIONAL SYSTEM: OPTIMAL CONTROL OF SUPPORT STRUCTURE
}

\author{
Mikhail Kharitonov ${ }^{1}$, Andrey Svetlov ${ }^{\S}$, Alexander Voronin ${ }^{3}$ \\ ${ }^{1,2,3}$ Mathematics and IT Department \\ Volgograd State University \\ pr. Universitetsky, 100, Volgograd, 400062, RUSSIA
}

\begin{abstract}
The article deals with the constrained dymanic optimization model of organization's operating core, which structure consists of the basic technological module and modules of support facilities. The production function of the operating core is represented as a superposition of Leontief's production functions corresponding to each of the modules.
\end{abstract}

AMS Subject Classification: 93B11, 90C05, 90C39, 90C70

Key Words: organizational system, operating core, optimization of the structure, production function, linear programming, optimal control, dynamic programming

\section{Introduction}

Papers $[7,8,9]$ propose a general approach to development and study of models for static and dynamic optimization of hierarchical structures with some "sectional" functions on them. These structures by the meaning fit with the structures of organizational systems (OS) control. In this paper we investigate a particular problem of this type with a special form of the organization's operational core structure and Leontief production function on it.

The traditional approach to synthesis of optimal mechanisms for OS control

Received: March 18, 2016

Published: May 5, 2016

$\S$ Correspondence author
(C) 2016 Academic Publications, Ltd.

url: www.acadpubl.eu 
[1] usually does not include the problem of optimizing the structure of production lines and factor flows. However, if a core technology is substantially regulated, the activity of the OS agents (employee or managers) is realized by minimizing of core technology's factor imbalances violations, that inevitably arise as a result of the institutional, informational and organizational uncertainty of OS. On the other hand, adhocracy organizations [3] have lack of core technologies, and for this reason production structure is more dependent on control mechanisms and conditions of the OS operation. Thus, formal models with dependence of production's factor structure on control tools allow to set and solve the optimal control problems for OS dynamics with a variable production and control structure.

\section{Structure-Dependent Production Function of an Organizational System}

To set optimization problem for the operating core of an OS we should construct a structure-dependent production function $(\mathrm{PF})$. As elements of PF (a simple transformers - ST) we use Leontief PF $F=k \min \left(f_{1} / a_{1}, \ldots, f_{n} / a_{n}\right)$, where $f_{i}$ indicate the values of transformation factors of production; $a_{1}, \ldots, a_{n}$ mean the technological coefficients; $k$ is the normalizing factor (fig. 1) [6]. Stable interfactor disproportions or fluctuations in transformation factors appreciably reduce the value of the $\mathrm{PF}$ of an $\mathrm{ST}$ (in comparison with its maximum). Within an OS, the flexibility of the $\mathrm{PF}$ with respect to its arguments is guaranteed through constructing a vertical structure of support facilities that produce the missing elements of transformation factors for the maximal efficiency of the basic ST. Therefore, the structure of factor production flows in the operating core of an OS can be expressed as a complex transformer (CT) - a multilevel structure of STs, where outputs of some STs represent inputs of the others. The structure of a CT acquires the form of an oriented graph, whose nodes are STs and edges correspond to factor flows. The last node is responsible for the basic production, whereas the rest nodes relate to support facilities.

Constructing the support ST and corresponding factor flows is an effective way to suppress an environmental uncertainty. It adds to PF of an OS some adaptive properties and induces an inner uncertainty to its dependence on the arguments, i.e. transformation factors. It's because the environment and inner parts of an OS determine the values of some parameters and uncertainties. That's why we should take into account all kinds of environmental and inner uncertainty during the optimization of the PF of an OS, as part of 


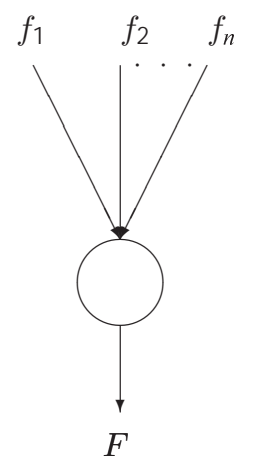

Figure 1: Simple transformer

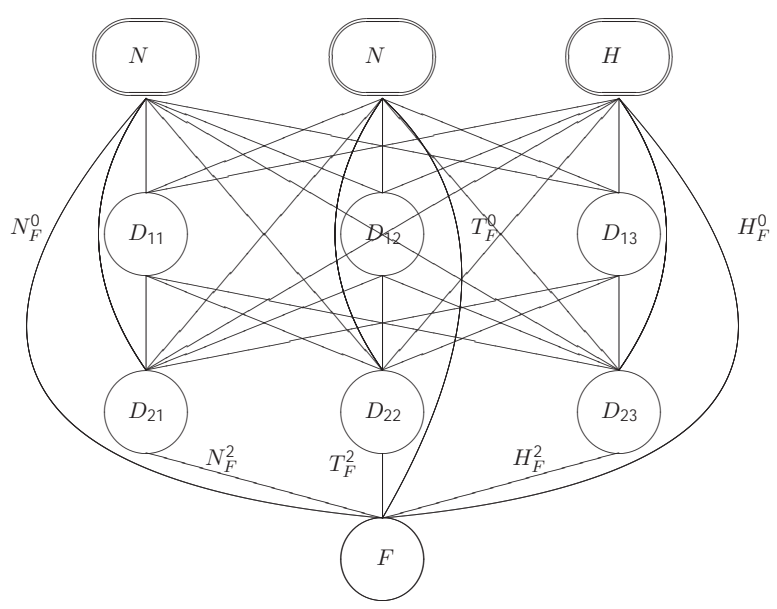

Figure 2: Two-level and three-factor complex transformer

an investigation of the optimal controls in an OS.

In this paper we consider the common structure of the CT with the highest state of factor aggregation. Papers [5, 6] deal with deterministic models (they take into account just an institutional uncertainty) of structure optimization for $\mathrm{CT}$ with restrictions on the production factors. Consideration of quality restrictions on the production factors as well as quantity ones leads us to the dependence of optimal structure of CT on value of reserves.

Figure 2 is an example of two-level and three-factor CT. In general case the numbers of levels and factors may be arbitrary. 


\section{Optimization Problem for Support Structure of the Operating Core of an Organizational System}

Let's consider a CT with a variable number of levels $n$ and three production factors $N, T, H$. System of equations (1)-(4) corresponds the deterministic optimization problem for CT structure, and it's similar to the one from [6]:

$$
\begin{gathered}
\sum_{l=1}^{n} \sum_{j=1}^{3} N_{l, j}^{0}+N_{F}^{0}=N ; \sum_{l=1}^{n} \sum_{j=1}^{3} T_{l, j}^{0}+T_{F}^{0}=T ; \sum_{l=1}^{n} \sum_{j=1}^{3} H_{l, j}^{0}+H_{F}^{0}=H \\
\sum_{k=i+1}^{n} \sum_{j=1}^{3} N_{k, j}^{i}=S_{l, 1} \min \left(\frac{1}{a_{l, 1}} \sum_{l=0}^{i-1} N_{i, 1}^{l}, \frac{1}{b_{l, 1}} \sum_{l=0}^{i-1} T_{i, 1}^{l}, \frac{1}{c_{l, 1}} \sum_{l=0}^{i-1} H_{i, 1}^{l}\right) \\
\sum_{k=i+1}^{n} \sum_{j=1}^{3} T_{k, j}^{i}=S_{l, 2} \min \left(\frac{1}{a_{l, 2}} \sum_{l=0}^{i-1} N_{i, 2}^{l}, \frac{1}{b_{l, 2}} \sum_{l=0}^{i-1} T_{i, 2}^{l}, \frac{1}{c_{l, 2}} \sum_{l=0}^{i-1} H_{i, 2}^{l}\right) \\
\sum_{k=i+1}^{n} \sum_{j=1}^{3} H_{k, j}^{i}=S_{l, 3} \min \left(\frac{1}{a_{l, 3}} \sum_{l=0}^{i-1} N_{i, 3}^{l}, \frac{1}{b_{l, 3}} \sum_{l=0}^{i-1} T_{i, 3}^{l}, \frac{1}{c_{l, 3}} \sum_{l=0}^{i-1} H_{i, 3}^{l}\right) \\
N_{F}^{n}=S_{n, 1} \min \left(\frac{1}{a_{n, 1}} \sum_{k=0}^{n-1} N_{n, 1}^{k}, \frac{1}{b_{n, 1}} \sum_{k=0}^{n-1} T_{n, 1}^{k}, \frac{1}{c_{n, 1}} \sum_{k=0}^{n-1} H_{n, 1}^{k}\right) \\
T_{F}^{n}=S_{n, 2} \min \left(\frac{1}{a_{n, 2}} \sum_{k=0}^{n-1} N_{n, 2}^{k}, \frac{1}{b_{n, 2}} \sum_{k=0}^{n-1} T_{n, 2}^{k}, \frac{1}{c_{n, 2}} \sum_{k=0}^{n-1} H_{n, 2}^{k}\right) \\
H_{F}^{n}=S_{n, 3} \min \left(\frac{1}{a_{n, 3}} \sum_{k=0}^{n-1} N_{n, 3}^{k}, \frac{1}{b_{n, 3}} \sum_{k=0}^{n-1} T_{n, 3}^{k}, \frac{1}{c_{n, 3}} \sum_{k=0}^{n-1} H_{n 3}^{k}\right)
\end{gathered}
$$

where $S_{i, j}=a_{i, j}+b_{i, j}+c_{i, j}$.

$$
F=(A+B+C) \min \left(\frac{N_{F}^{n}+N_{F}^{0}}{A}, \frac{T_{F}^{n}+T_{F}^{0}}{B}, \frac{H_{F}^{n}+H_{F}^{0}}{C}\right)
$$

Three equations (1) describe the distribution of the original production factors by the structure of the CT. The next three equations (2) gives the balance expressions for nodes $D_{i 1}, D_{i 2}, D_{i 3}$ respectively $(i=\overline{1, n})$. Three equations (3) correspond to the last support ST $(i=n) . N_{j, l}^{i}$ denotes the value of the factor $N$ produced by ST $i$ and applied by ST $j$ ( $l$ is the index of the production factor).

Take the variables of the system (1)-(4) and compile the factor flow vector $\varphi$ of the CT. 
Under fixed $N, T N, H N$ the optimization problem for the PF of the CT (1)(4) with variable structure takes the form

$$
F=(A+B+C) \min \left(\frac{N_{F}^{n}+N_{F}^{0}}{A}, \frac{T_{F}^{n}+T_{F}^{0}}{B}, \frac{H_{F}^{n}+H_{F}^{0}}{C}\right) \rightarrow \max _{\varphi, n}
$$

Note that the problem (5) is equivalent to the following linear programming (LP) problem:

$$
\begin{array}{r}
-\frac{A+B+C}{A}\left(N_{F}^{n}+N_{F}^{0}\right)+F \leq 0, \\
-\frac{A+B+C}{B}\left(T_{F}^{n}+T_{F}^{0}\right)+F \leq 0, \\
-\frac{A+B+C}{C}\left(H_{F}^{n}+H_{F}^{0}\right)+F \leq 0, \\
F \rightarrow \max _{N, T, H} .
\end{array}
$$

Similarly, the problem (1)-(5) gets reduced to the parametric LP problem

$$
\begin{aligned}
& \varphi_{p}(n) \rightarrow \max _{n, \varphi}, \\
& \sum_{j=1}^{p(n)} M_{i j} \varphi_{j} \leq \beta_{i}, i=\overline{1, u(n)}, \\
& p(n)=\frac{n^{2}+5 n+8}{2}, u(n)=3 n+6 .
\end{aligned}
$$

Here $p(n)$ means the number of variables and $u(n)$ indicates the number of inequalities.

Specifically, if $n=1, A=B=C=a_{i}=b_{i}=c_{i}=1$, we obtain 


$$
\varphi=\left(\begin{array}{cccccccccc}
1 & 1 & 1 & 0 & 0 & 0 & 0 & 0 & 0 & 0 \\
0 & 0 & 1 & 0 & 0 & 0 & & & & \\
0 & 0 & 0 & 1 & 1 & 1 & 0 & 0 & 0 & 0 \\
0 & 0 & 0 & 1 & 0 & 0 & & & & \\
0 & 0 & 0 & 0 & 0 & 0 & 1 & 1 & 1 & 0 \\
0 & 0 & 0 & 0 & 1 & 0 & & & & \\
0 & 0 & 0 & 0 & 0 & 0 & 0 & 0 & 0 & -3 \\
0 & 0 & -3 & 0 & 0 & 1 & & & & \\
0 & 0 & 0 & 0 & 0 & 0 & 0 & 0 & 0 & 0 \\
-3 & 0 & 0 & -3 & 0 & 1 & & & & \\
0 & 0 & 0 & 0 & 0 & 0 & 0 & 0 & 0 & 0 \\
0 & -3 & 0 & 0 & -3 & 1 & & & & \\
-3 & 0 & 0 & 0 & 0 & 0 & 0 & 0 & 0 & 1 \\
0 & 0 & 0 & 0 & 0 & 0 & & & & \\
0 & 0 & 0 & -3 & 0 & 0 & 0 & 0 & 0 & 1 \\
0 & 0 & 0 & 0 & 0 & 0 & & & & \\
0 & 0 & 0 & 0 & 0 & 0 & -3 & 0 & 0 & 1 \\
0 & 0 & 0 & 0 & 0 & 0 & & & & \\
0 & -3 & 0 & 0 & 0 & 0 & 0 & 0 & 0 & 0 \\
1 & 0 & 0 & 0 & 0 & 0 & & & & \\
0 & 0 & 0 & 0 & -3 & 0 & 0 & 0 & 0 & 0 \\
1 & 0 & 0 & 0 & 0 & 0 & & & & \\
0 & 0 & 0 & 0 & 0 & 0 & 0 & -3 & 0 & 0 \\
1 & 0 & 0 & 0 & 0 & 0 & & & & \\
0 & 0 & -3 & 0 & 0 & 0 & 0 & 0 & 0 & 0 \\
0 & 1 & 0 & 0 & 0 & 0 & & & & \\
0 & 0 & 0 & 0 & 0 & -3 & 0 & 0 & 0 & 0 \\
0 & 1 & 0 & 0 & 0 & 0 & & & & \\
0 & 0 & 0 & 0 & 0 & 0 & 0 & 0 & -3 & 0 \\
0 & 1 & 0 & 0 & 0 & 0 & & & &
\end{array}\right),
$$

Right side vector has form $\beta=(N, T, H, 0, \ldots, 0)$. Let $X \geq \max (Y, Z)$, then $\zeta=1.0$, and $0 \leq \xi \leq 1.0,0 \leq \eta \leq 1.0$.

Next we consider scalable values of variables. Denote

$$
\zeta=\frac{N}{\max (N, T, H)}, \quad \xi=\frac{T}{\max (N, T, H)}, \quad \eta=\frac{H}{\max (N, T, H)} .
$$


a)

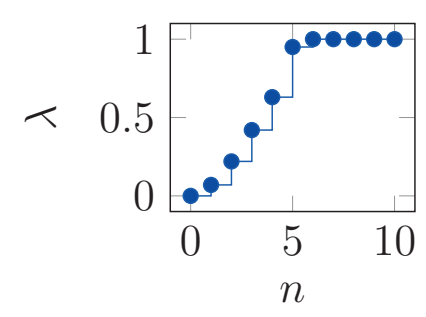

c)

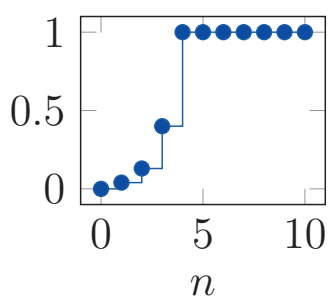

d)

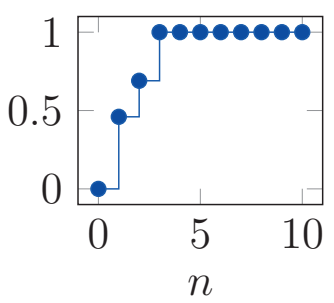

Figure 3: The curve of dependences of $\lambda$ on number of levels $n, K=$ $[1,5]$ with a) $\xi=0.01, \eta=0.2$; b) $\xi=0.01, \eta=1.0$; d ) $\xi=0.1, \eta=0.2$;

Now we set $\beta=(1, \xi, \eta, 0,0, \ldots, 0)$.

Let's $\varphi_{p}^{*}(n)$ is the solution of problem (6), and the optimal structure of the CT described by the system (1)-(5) is the quantity $n^{*}=\min _{n}\left(\arg \max _{n} \varphi_{p}^{*}(n)\right)$.

There is an algorithm for automatic inequality (6) development that used for investigation the dependence of problem (6) solution on vector parameter with various $n$.

Denote $\lambda=F /(1+\xi+\eta)$. External parameters of problem (6) are proportions of input variables $1: \xi: \eta$ and proportions of technological coefficients $a_{i}: b_{i}: c_{i}$. For each $\mathrm{CT}$, the quantities $a_{i}, b_{i}, c_{i}$ are assumed given. Numerical experiments employ random realizations of these parameters; their values were fixed while varying the number of levels. Here is the results on solutions of two specific problems (6):

(6)(a): problem (6) with fixed inputs.

(6)(b): problem (6) with fixed $n$.

Figure 3 shows the results on solution parametric problem (6) (a) by simplex method [4] for some values $\xi, \eta$ and random values of paremeters $a_{i}, b_{i}, c_{i}$ from segment $K \in R^{+}$.

Figure 4 shows some solutions of problem(6) (b): graph of variance $\lambda$ with $\xi \in[0,1], \eta \in[0,1]$, step $\Delta=0.1$, and $n=\overline{1,4}$. 

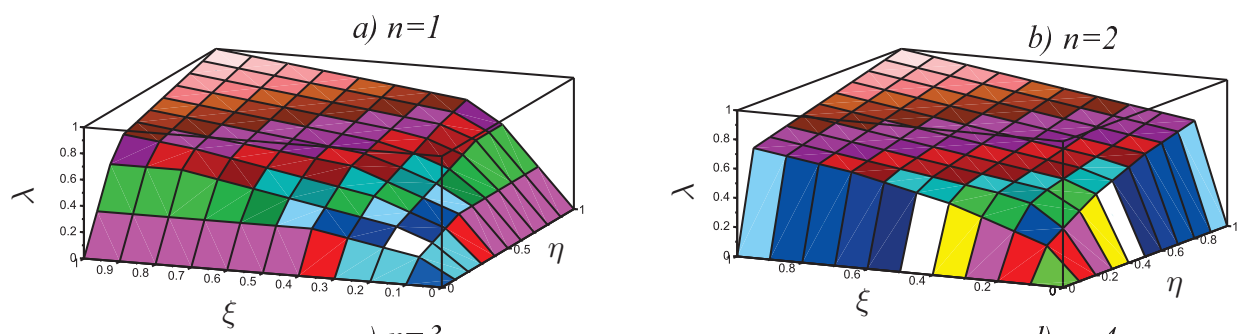

c) $n=3$
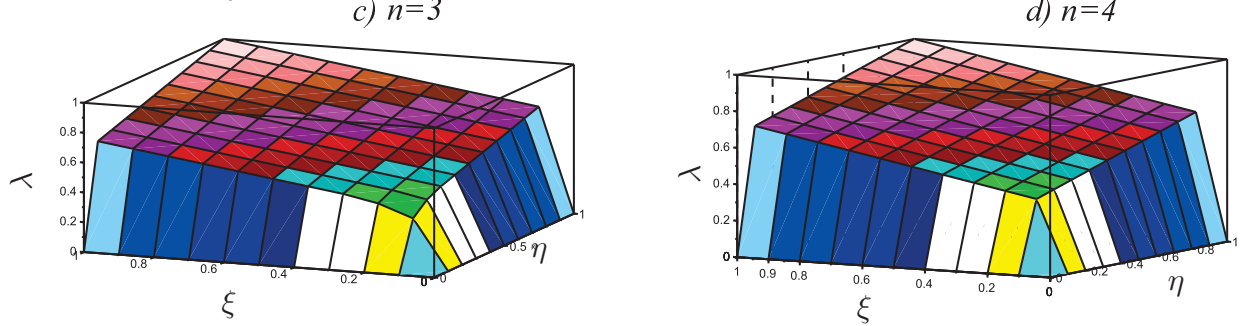

Figure 4: The curve of $F(1, \xi, \eta)$ with $n=\overline{1,4}$

\section{Dynamic Optimization of Support Facilities of the Organizational Structure's Operating Core}

Problem (6) as above is a part of structure dynamic optimization of OS's operating core with given time series of production factors values. It involves finding optimal time series of OC's structure which maximize OS's income on some controlled time interval. Here is formal problem setting.

Let's $\Phi(n(t))$ is a value of the PF of the OC (solution of the problem (6)) at the moment $t$ with $n(t)$ and given production factors values $\xi(t), \eta(t)$; $\gamma$ is an added cost coefficient. Expenses on the OS's operating core control consist of constants $Q(n(t))$ proportional to number of ST, and variables $W(n(t))$ proportional to number of non-zero factor flows in $n(t)$-levels structure of the OC. Thus the expenses on the OC control at the moment $t$ have form $\Xi(n(t))=\beta[W(n(t))+Q(n(t))]$, where $\beta$ is some constant.

Each change of value $n$ leads to expenses on construction some new structural bonds and destruction some old ones. Assume for simplicity that function of expenses on reconstruction the OC structure has form

$$
U(t)=\alpha[n(t+1)-n(t)]
$$

where $\alpha$ is some constant. 
a)

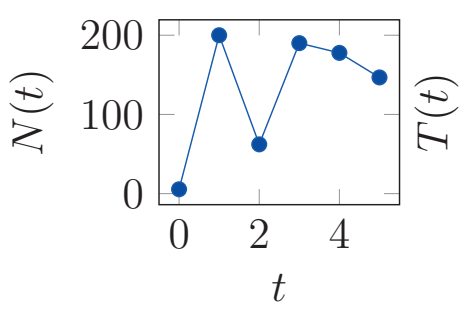

b)

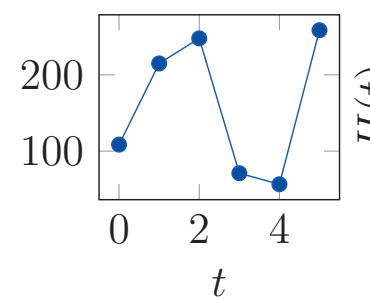

c)

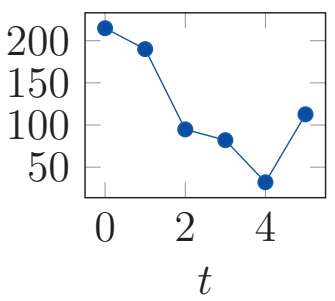

Figure 5: Time series of production factors

Dynamic optimization problem for OS's OC with $t \in[0, T]$ has form:

$$
J=\sum_{t=0}^{T-1} J(n(t), t)+\hat{J}(n(T), T) \rightarrow \max _{n(t), t \in[0, T]}
$$

where $J(n(t), t)=\gamma \Phi(n(t))-U(t)-\Xi(n(t)) ; \hat{J}(n(T), T)=\gamma \Phi(n(T))-\Xi(n(T))$.

We solve problem (7) numerically by the dynamic programming method (similar to [5]).

Here is for instance the solution of the problem (7) with $T=5$, technological coefficients are equal to one, production factors are given by the time series from the Fig. 5. We call this example the problem (7.a)

The solution of the problem (7.a) falls into independend problems (6) with each value of $t$ (degenerate problem (7.a.0)) and has form $n^{*}(t)=(4,1,2,2,2,3)$ (Fig. $6 \mathrm{f})$ ). Form of target function for the problem $(7)$ shows that its actual parameters are $\xi=\frac{\alpha}{\gamma}$ and $\eta=\frac{\beta}{\gamma}$.

Let's consider two integral parameters for solutions of the problem (7):

$$
\mu=\frac{1}{T} \sum_{t=0}^{T} n^{*}(t), \delta=\frac{1}{T} \sum_{t=0}^{T-1}\left|n^{*}(t+1)-n^{*}(t)\right|
$$

They describe mean complexity and mean variance of the OC structure.

Thus each optimal curve $n^{*}(t)$ corresponds the vector parameter $p=(\xi, \eta, \mu$, $\delta)$.

After analysis of the vector parameter $p=(\xi, \eta, \mu, \delta)$ values for optimal curves $n^{*}(t)$ we can mark out zones on the plane $(\xi, \eta)$, which corresponds to 
some common dynamic modes. Zones boundaries are defined by parameters $\left(\mu_{0}, \delta_{0}\right)$ of degenerate problem (7.0) (problem (7) with $\left.\alpha=\beta=0, \gamma>0\right)$. As the common conditions modes we can mark out the following ones:

- Dynamic modes $(\delta>0)$ :

1. dynamic tracking mode (optimal curve $n^{*}(t)$ here coincides with the same curve of degenerate problem (7.0))

(I, $\mu=\mu_{0}, \delta=\delta_{0}$ ).

2. dynamic mode with a simplified OC structure

(II, $\mu<\mu_{0}, \delta \leq \delta_{0}$ ).

3. dynamic mode with a complicated OC structure

(III, $\mu>\mu_{0}, \delta \leq \delta_{0}$ ).

- Stationary modes:

1. mode with the simplest OC structure $\left(\delta=\frac{\left|n^{*}(1)-n^{*}(0)\right|}{T}\right)$

$\left(\mathrm{IV}, n\left(t_{0}\right)=n_{0}, n^{*}(t)=1, \forall t>t_{0}\right)$.

2. mode with an initial OC structure $(\delta=0)$

$\left(\mathrm{V}, n^{*}(t)=n_{0}\right)$.

Value of $\delta$ in zones II and III decreases with distance from the origin in any direction.

Fig. 6 a)-e) show some solutions of the problem (7.1).

\section{Conclusion}

The results of numerical simulation of the structural optimization model for the operating core have testified to the following: considerable reserves of the factors in support facilities and a sufficiently high number of structure levels of operating core of an OS allow to achieve the stable maximum of the PF under appreciable deviations in the proportions of the basic production (from their optimal values) and fluctuations in the technological coefficients of support facilities ST. The increasing factor disproportion and variation of the technological coefficients of support facilities ST gets compensated by the growing value of production factors reserves and complexity of the OC structure.

Substantive interpretation of vector $(\xi, \eta)$ allows to refer the common dynamic modes to Mintzberg's five types of organizational structure as follows: 
a)

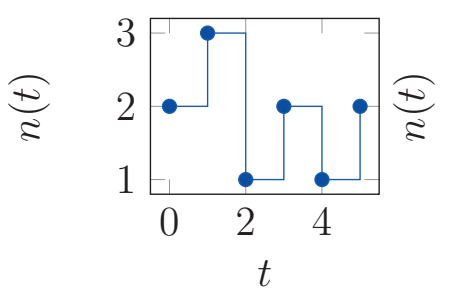

d)

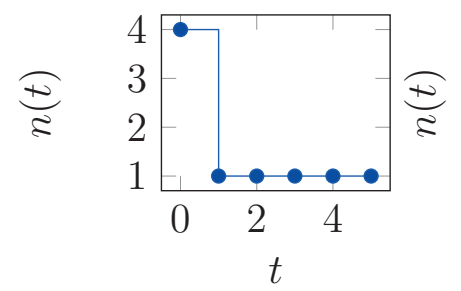

b)

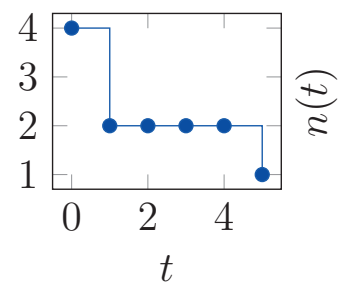

e)

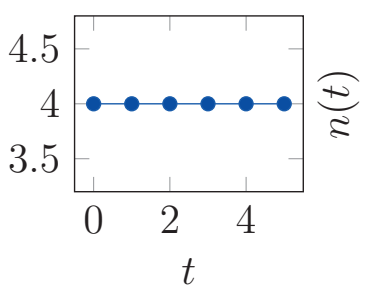

c)

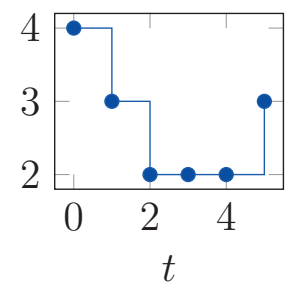

f)

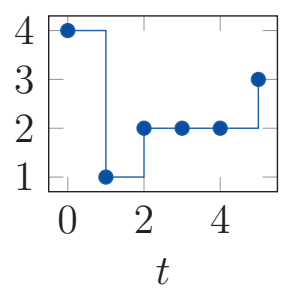

Figure 6: Optimal curves $n(t)$ - solutions of the problem (7.a). a) $p=(50,100,2.8,1.0), I$; b) $p=(250,200,2.6,1.0), I I$; c) $p=$ $(600,50,3.2,0.6), I I I$, d) $p=(100,800,1.8,0.60), I V$, e) $p=$ $(750,0,4.8,0.0), V ., \quad$ f) Solution of the problem (7.a.0) $(p=$ $(0.0,0.0,2.8,1.0))$

I - adhocracy. The OC structure for this type of OS is sensitive to external changes. Such OSs are often associated with innovation.

II - divisional structure. This type of OS functions best if conditions are not too complicated and not too dynamic.

III - professional bureaucracy. In the OC of this OS type highly qualified professionals dominate. They operate difficult, but clearly stipulated procedures.

IV — simple structure. OSs of this type are generally young, small or crisis ones.

$\mathrm{V}$ - mechanistic bureaucracy. OSs of this type are generally large and mature ones. It operates in a stable environment.

To develop and correct the formalization of Mintzberg's theory of organization structure we need to integrate the proposed here models with models of 


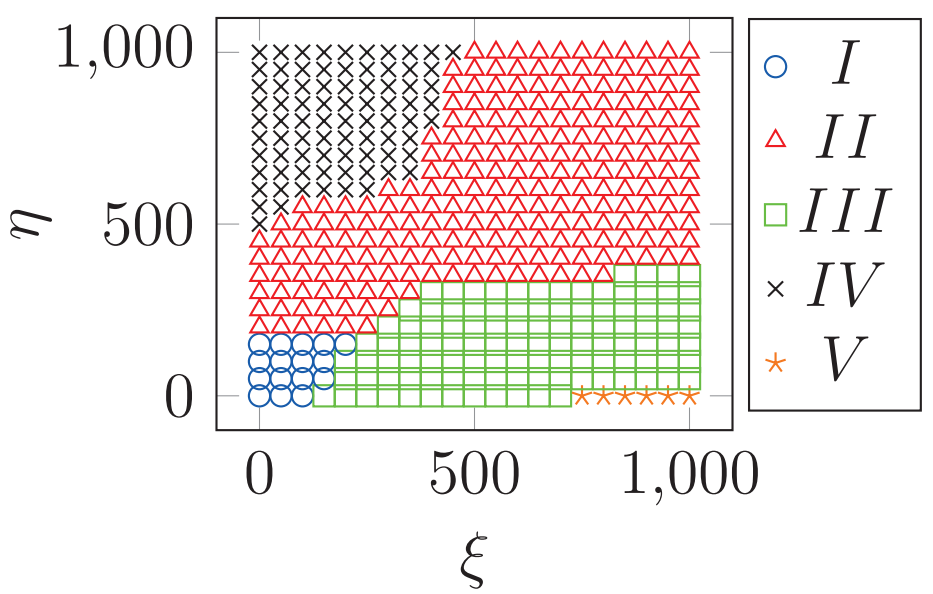

Figure 7: Common dynamic modes zones for the problem (7.1).

optimal control hierarchies (based on the models of $[7,8]$ ) and models of an OS control tools [1].

\section{Acknowledgements}

The study was supported by RFBR, project 16-48-340147-r_a.

\section{References}

[1] Burkov V. N., Goubko M., Korgin N., Novikov D. Introduction to Theory of Control in Organizations, Boca Raton: CRC Press, USA (2015).

[2] Kleiner G.B. Production functions. Theory, methods and application, Finance and Statistics, Russia (1986) (in Russian).

[3] Mintzberg H. Structure in Fives: Designing Effective Organizations, Prentice Hall, USA (1992).

[4] Papadimitriou C., Steiglitz K. Combinatorial optimization: Algorithms and complexity, Prentice Hall, USA (1982).

[5] Voronin A.A., Kharitonov M.A. Constrained dynamic optimization model of organization's operating core, Vestnik VolGU, 17, 2 (2012), 41-59 (in Russian). DOI: 10.15688/jvolsu1.2012.2.6.

[6] Voronin A.A., Kharitonov M.A. The operating core of an organization: a constrained optimization model, Automation and Remote Control, 75, 1 (2014), 167-178. DOI: 10.1134/S0005117914010135. 
[7] Voronin A.A., Mishin S.P. Algorithms to seek the optimal structure of the organizational system, Automation and Remote Control, 63, 5 (2002), 803-814. DOI: 10.1023/A:1015454106119.

[8] Voronin A.A., Mishin S.P. A model of optimal control of structural changes in an organizational system, Automation and Remote Control, 63, 8 (2002), 1329-1342. DOI: 10.1023/A:1019839612227.

[9] Voronin A.A., Mishin S.P. Organization system structure modeling. About optimum tree search algorithms, Vestnik VolGU , 6, (2001), 78 (in Russian). 
